TRANSACTIONS OF THE

AMERICAN MATHEMATICAL SOCIETY

Volume 356, Number 4, Pages 1443-1462

S 0002-9947(03)03356-7

Article electronically published on September 22, 2003

\title{
EXTENSION OF CR-FUNCTIONS INTO WEIGHTED WEDGES THROUGH FAMILIES OF NONSMOOTH ANALYTIC DISCS
}

\author{
DMITRI ZAITSEV AND GIUSEPPE ZAMPIERI
}

\begin{abstract}
The goal of this paper is to develop a theory of nonsmooth analytic discs attached to domains with Lipschitz boundary in real submanifolds of $\mathbb{C}^{n}$. We then apply this technique to establish a propagation principle for wedge extendibility of CR-functions on these domains along CR-curves and along boundaries of attached analytic discs. The technique from this paper has been also extensively used by the authors recently to obtain sharp results on wedge extension of CR-functions on wedges in prescribed directions extending results of Boggess-Polking and EAstwood-Graham.
\end{abstract}

\section{INTRODUCTION}

The classical HARTOGS extension theorem states that holomorphic functions in a neighborhood of the Hartogs figure extend holomorphically to a larger subset. Such automatic extension to larger subsets plays an important role in the study of holomorphic functions in several complex variables. A general result of this type has been recently obtained by Tumanov [T95]. He shows, for a wedge $V$ with generic edge $E$ in a submanifold $M \subset \mathbb{C}^{n}$, that holomorphic functions in a (fixed) neighborhood of $V$ extend holomorphically to a (fixed) wedge in $\mathbb{C}^{n}$ with edge $E$.

If the edge $E$ is not generic, the conclusion about joint extension to a wedge may not hold (see Example 2.3 below). The same may happen if the edge $E$ is not smooth but only Lipschitz (see Example 2.4). However, one may still ask:

When do all holomorphic functions in a neighborhood of $V$ extend holomorphically to a subset of a given geometric shape near $E$, not necessarily a wedge?

In this paper we give conditions on $V$ that guarantee holomorphic extension in the above sense to regions more general than wedges which we call $\alpha$-wedges and which can be viewed as wedges in the space where the normal directions to $M$ have a weight $0<\alpha<1$ (see Definition 2.2). In order to treat equally the case of wedges with Lipschitz edges, we consider a more general class of open sets $V \subset M$ with Lipschitz boundary, and for every $p \in \partial V$ we denote by $C_{p} V \subset T_{p} M$ the open tangent cone (see 92). We define the complex angle of $V$ at $p$ to be the maximal angle of the intersection of $C_{p} V$ with a complex line in $T_{p} M$. If all intersections are empty, we say that the complex angle is 0 . It is clear that the complex angle is a local biholomorphic invariant of $V$ at $p$.

Received by the editors July 25, 2002.

2000 Mathematics Subject Classification. Primary 32V10, 32V25, 32D15.

(C)2003 American Mathematical Society 
We prove:

Theorem 1.1. Let $M \subset \mathbb{C}^{n}$ be a generic submanifold of class $C^{4}$ and $V \subset M$ an open subset with Lipschitz boundary at a point $p \in \partial V$ with complex angle $\pi \alpha$ for some $\alpha>1 / 2$. Then for every neighborhood $V^{\prime}$ of $V$ in $\mathbb{C}^{n}$ there exists an $\alpha$-wedge $V^{\prime \prime}$ in $\mathbb{C}^{n}$ over $V$ at $p$ such that all holomorphic functions in $V^{\prime}$ extend holomorphically to $V^{\prime \prime}$.

The angle $\pi \alpha=\pi / 2$ plays a special role, as we observed in ZZ01. The main reason is that for $\alpha>1 / 2$ and $t>0$ small, the power $t^{1 / \alpha}$ dominates the power $t^{2}$ that appears in the defining equations of $M$. Observe that in the result of T95] mentioned above, where $V$ is a wedge with generic edge $E$, the complex angle of $V$ at a point $p \in E$ is always $\pi$ (see [ZZ01]), and hence $\alpha>1 / 2$. In contrast to [T95], the edge $E$ plays a secondary role in Theorem 1.1 and can be seen as a subset of the Lipschitz boundary of $V$.

The result of TUMANOV [T95] was obtained as a consequence of a more general propagation principle for wedge-extendibility of CR-functions that we also establish here in our case. Recall that a CR-curve in $M$ is a piecewise smooth curve $\gamma:[0,1] \rightarrow M$ with $\gamma^{\prime}(t) \in T_{\gamma(t)}^{c} M$ for all $t \in[0,1]$, where $T_{x}^{c} M:=T_{x} M \cap i T_{x} M$. We have:

Theorem 1.2 (Propagation of wedge extension along CR-curves). Let $M \subset \mathbb{C}^{n}$ be a generic submanifold of class $C^{4}$, and $V \subset M$ an open subset with Lipschitz boundary at a point $p \in \partial V$. Let $\gamma:[0,1] \rightarrow\{p\} \cup V$ be a CR-curve with $\gamma(0)=p$ such that $\gamma^{\prime}(0) \in C_{p} V$ and the angle of the sector $C_{p} V \cap \mathbb{C} \gamma^{\prime}(0)$ is $\pi \alpha$ for some $1 / 2<\alpha<1$. Then, for any wedge $V^{\prime}$ with edge $V$ in $\mathbb{C}^{n}$ at $\gamma(1)$, there exists an $\alpha$-wedge $V^{\prime \prime}$ in $\mathbb{C}^{n}$ over $V$ at $p$ such that any continuous $C R$-function on $V$ that has a holomorphic extension to $V^{\prime}$ also has a holomorphic extension to $V^{\prime \prime}$.

As in T95] we also study extension of CR-functions to wedges in submanifolds of lower dimension:

Theorem 1.3 (Propagation of CR-extension along CR-curves). Under the assumptions of Theorem 1.2, for any wedge $V^{\prime}$ with edge $V$ at $\gamma(1)$ in a submanifold of $\mathbb{C}^{n}$ with boundary $V$, there exists an $\alpha$-wedge $V^{\prime \prime}$ over $V$ at $p$ in a submanifold of $\mathbb{C}^{n}$ with boundary $M$ such that any continuous CR-function on $V$ that has a CR-extension to $V^{\prime}$ also has a CR-extension to $V^{\prime \prime}$.

As in [T95] we obtain Theorems 1.2 and 1.3 by approximating a CR-curve by a sequence of attached analytic discs and applying a propagation result along such discs. The main difference here is that we allow nonsmooth discs $A: \bar{\Delta} \rightarrow \mathbb{C}^{n}$ of the form

$$
A(\tau)=(1-\tau)^{\alpha} A_{0}+A_{1}(\tau)
$$

where $A_{0} \in \mathbb{C}$ and $A_{1} \in C^{1, \beta}(\bar{\Delta}) \cap \mathcal{O}(\Delta)$ for some $0<\beta<1$. By a small disc of the above form we mean a disc with both $A_{0}$ and $A_{1}$ being small in corresponding norms. Our propagation result along analytic discs can be stated as follows:

Theorem 1.4 (Propagation along nonsmooth analytic discs). Let $M \subset \mathbb{C}^{n}$ be a generic submanifold of class $C^{4}$ through $p=0$, and $V \subset M$ an open subset with Lipschitz boundary at $p \in \partial V$. Fix a vector $v \in T_{p} M$ such that, for some $1 / 2<\alpha<1$, the sector $\left\{\tau^{\alpha} v: \operatorname{Re} \tau \geq 0, \tau \neq 0\right\}$ is contained in $C_{p} V$. Then, for 
any sufficiently small analytic disc $A$ attached to $M$ of the form (1) with $A_{0} \in \mathbb{R}_{+} v$, $A_{1} \in C^{1, \beta}(\bar{\Delta})$ and $A_{1}(1)=p$, and for any $q \in A(\partial \Delta) \cap V$, the following hold:

(i) For any wedge $V^{\prime} \subset \mathbb{C}^{n}$ with edge $V$ at $q$, there exists an $\alpha$-wedge $V^{\prime \prime} \subset$ $\mathbb{C}^{n}$ at $p$ over $V$ such that any continuous CR-function on $V$ that has a holomorphic extension to $V^{\prime}$ also has a holomorphic extension to $V^{\prime \prime}$.

(ii) For any wedge $V^{\prime}$ with edge $V$ at $q$ in a submanifold $M^{\prime}$ with boundary $V$ of class $C^{4}$, there exists an $\alpha$-wedge $V^{\prime \prime}$ over $V$ at 0 in a submanifold $M^{\prime \prime} \subset \mathbb{C}^{n}$ with boundary $M$ of class $C^{1}$ such that any continuous $C R$-function on $V$ that has a CR-extension to $V^{\prime}$ also has a CR-extension to $V^{\prime \prime}$. Moreover, given several wedges $V_{1}^{\prime}, \ldots, V_{s}^{\prime}$ as above in s linearly independent directions in $T_{q} \mathbb{C}^{n} / T_{q} M$, the corresponding submanifolds $M_{1}^{\prime \prime}, \ldots, M_{s}^{\prime \prime}$ can be chosen in s linearly independent directions in $T_{p} \mathbb{C}^{n} / T_{p} M$.

Theorems 1.21 .4 can be seen as boundary versions of corresponding propagation results for interior points of a generic submanifold due to HANGES-SJÖSTRAND [HS82], Hanges-Treves [HT83], Trépreau [Tr90] and Tumanov [T93], T94].

We now compare our methods with those of [T95. The assumption that $V$ is a wedge with generic edge $E$ was essentially used in [T95] for the construction of smooth $\left(C^{1, \alpha}\right)$ analytic discs $A: \bar{\Delta} \rightarrow \mathbb{C}^{n}$ attached to $\bar{V}$ for which open pieces of $A(\partial \Delta)$ are contained in $E$. The assumption that the edge has regularity at least $C^{1, \alpha}$ was also needed there. In our situation such discs may not exist. Our approach is based on attaching nonsmooth analytic discs $A$ as in (II) for which $A(1) \in \partial V$ and $A(\partial \Delta \backslash\{1\}) \subset V$. In particular, the case of Lipschitz edges can also be treated. The next technical difficulty arising here when pursuing known methods is to determine what should be the directions of those discs at their singular points. The classical theory of attaching discs in Hölder spaces $C^{\alpha}$ is not satisfactory, since a general Hölder vector-function may not be differentiable and hence its direction at a boundary point may not be defined in a reasonable sense. Instead of working in this large class we solve the Bishop's equation in a more restrictive class of " $\alpha$ discs" of the form (1) for which their directions are defined in the space normal to $A_{0}$ and are given by the derivatives of $A_{1}$. The $\alpha$-wedges are obtained as unions of those discs as $A(1)$ moves in $V$.

Another important feature of our method is that all normal directions to $M$ for an $\alpha$-wedge are obtained directly by deforming $\alpha$-discs rather than by separately treating directions of automatic extension and directions transversal to them as in [T95.

Finally, establishing uniform approximation of CR-functions on $V$ by polynomials in the spirit of the celebrated BAOUENDI-TREVEs theorem [BT81] requires additional care in our case. Since the boundary $\partial V$ may not contain generic submanifolds, the method of [BT81] cannot be applied directly as in T95. Here we refine the arguments to obtain approximation separately on the boundary of each sufficiently small $\alpha$-disc; this suffices for our purposes.

We conclude by giving an application of Theorem 1.2 to a description of holomorphic hulls of pieces of generic real submanifolds near their boundaries. Recall that a generic submanifold $M \subset \mathbb{C}^{n}$ is called minimal at a point $q \in M$ if any submanifold $S \subset M$ through $q$ with $T_{x} S \supset T_{x}^{c} M$ for all $x \in S$ coincides with $M$. By Tumanov's extension theorem [T88], [T90], if $M$ is minimal at $q$, all CR-functions on $M$ extend holomorphically to a wedge in $\mathbb{C}^{n}$ with edge $M$ at $q$. Hence we obtain the following immediate corollary of Theorem 1.2 
Corollary 1.5. Let $M, V, p, \alpha$ and $\gamma$ be as in Theorem 1.2 and suppose in addition that $M$ is minimal at $\gamma(1)$. Then all continuous $C R$-functions on $V$ extend holomorphically to an $\alpha$-wedge in $\mathbb{C}^{n}$ at $p$ over $V$.

It follows that also polynomial, rational and holomorphic (with respect to a Stein neighborhood) hulls of $V$ contain an $\alpha$-wedge in $\mathbb{C}^{n}$ at $p$ over $V$.

\section{Preliminaries AND EXAmples}

In this section we recall some basic concepts used in $\$ 1$ and conclude by giving examples demonstrating phenomena that were discussed there.

By a cone $\Gamma$ in the euclidean space $\mathbb{R}^{m}$ we always mean a subset invariant under multiplications by positive real numbers. A subcone $\Gamma^{\prime} \subset \Gamma$ is said to be strictly smaller if $\overline{\Gamma^{\prime}} \backslash\{0\}$ is contained in the interior of $\Gamma$. We say that an open subset $V$ in a $\left(C^{1}\right)$ manifold $M$ has Lipschitz boundary at a point $p \in \partial V$ if, in suitable coordinates near $p, \partial V$ is be represented by the graph of a Lipschitz function. It is easy to see that $V$ has Lipschitz boundary at $p$ if and only if there exists an open cone $\Gamma \subset T_{p} M$ such that, for any strictly smaller subcone $\Gamma^{\prime} \subset \Gamma$ and some (and hence any) local coordinates on $M$ in a neighborhood of $p$, one has $x+y \in V$ for all $x \in V$ and $y \in \Gamma^{\prime}$ sufficiently close to $p$ and 0 respectively (here the sum is taken with respect to the given coordinates). It is clear that, if $V$ satisfies the above property with respect to two cones $\Gamma_{1}, \Gamma_{2} \subset T_{p} M$, it also does with respect to their sum $\Gamma_{1}+\Gamma_{2}$. Furthermore, among all such cones there is a unique maximal one, namely the sum of all of them, which is automatically convex. We call it the tangent cone to $V$ at $p$, and denote it by $C_{p} V$.

The notion of Lipschitz boundary is convenient for defining wedges in a slightly more general context than was used in [T95]:

Definition 2.1. Let $M \subset \mathbb{R}^{m}$ be a submanifold and $p \in M$. A wedge with edge $M$ at $p$ is an open subset in $\mathbb{R}^{m}$ with Lipschitz boundary at $p \in \partial V$ such that the boundary $\partial V$ contains a neighborhood of $p$ in $M$.

Our next basic notion is that of an $\alpha$-wedge:

Definition 2.2. Let $M \subset \mathbb{R}^{m}$ be a submanifold, $V \subset M$ an open subset and $p \in \partial V$. Fix $0<\alpha<1$. An $\alpha$-wedge in $\mathbb{R}^{m}$ over $V$ at $p$ is an open subset $V^{\prime} \subset \mathbb{R}^{m}$ for which there exist a neighborhood $\Omega$ of $p$ in $\mathbb{R}^{m}$, a wedge $W$ with edge $M$ at $p$ and a constant $C>0$ such that

$$
V^{\prime} \cap \Omega \supset\left\{x \in W: \operatorname{dist}(x, V)<C \operatorname{dist}(x, \partial V)^{1 / \alpha}\right\},
$$

where the distance is taken with respect to any Riemannian metric.

If $M^{\prime} \subset \mathbb{R}^{m}$ is another submanifold containing $M$, both wedges and $\alpha$-wedges in $M^{\prime}$ are defined in the obvious way with respect to local coordinates on $M^{\prime}$. Both notions also make sense if $M^{\prime}$ is a manifold with boundary $M$ at a point $p \in M$.

In our situation $M$ will always be a generic submanifold of $\mathbb{C}^{n}$, i.e. $T_{x} M+$ $i T_{x} M=T_{x} \mathbb{C}^{n}$ for all $x \in M$. The CR-functions on $M$ are defined as functions satisfying tangential Cauchy-Riemann equations in the distributional sense. In the sequel, CR-functions will be assumed at least continuous, i.e. of class $C^{0}$. In all our results, the extension of CR-functions of any more restrictive class $C^{s}(1 \leq s \leq \infty)$ is also obtained in the same class $C^{s}$.

The following elementary example shows that, if the edge is not generic, a domain of holomorphy containing the wedge may not contain any $\alpha$-wedge for any $\alpha$. 
Example 2.3. Consider the hyperplane $M:=\left\{\operatorname{Im} z_{2}=0\right\}$ in $\mathbb{C}_{z_{1}, z_{2}}^{2}$ and the wedge (half-space) $V:=\left\{z \in M: \operatorname{Re} z_{2}>0\right\}$ with edge $E:=\left\{z \in M: z_{2}=0\right\}$. Then for any domain $D \subset \mathbb{C}$ containing $\mathbb{R}_{+}$there exist holomorphic functions in $\mathbb{C} \times D \supset V$ that do not extend holomorphically to any larger domain.

The following example shows that the conclusion of [T95] may not hold for wedges with Lipschitz edges.

Example 2.4. Consider the hypersurface $M:=\left\{z=\left(z_{1}, z_{2}\right) \in \mathbb{C}^{2}: \operatorname{Im} z_{2}=\left|z_{1}\right|^{2}\right\}$ and, for a real number $1 / 2<\alpha<1$, the wedge $V:=\left\{z \in M: \operatorname{Im} z_{2}<\operatorname{Im} z_{1}^{1 / \alpha}\right\}$ with edge $E:=\left\{z \in M: z_{1}=0\right\}$ at 0 . Here the edge is not generic. We can also view $V$ as a wedge with edge $\widetilde{E}:=\left\{z \in M: \operatorname{Im} z_{2}=\operatorname{Im} z_{1}^{1 / \alpha}\right\}$ that is generic and Lipschitz (i.e. can be locally represented as a graph of a Lipschitz function) but not smooth.

We claim that the CR-functions on $V$ do not extend holomorphically to any wedge $W$ with edge $E$ at 0 . Indeed, any such wedge $W$ must be contained in the convex hull of $V$ and hence must intersect one of the analytic discs $A_{c}(\tau):=$ $\left(\tau, c \tau^{1 / \alpha}\right), c \geq 1$, defined for $\tau \in \mathbb{C}$ with $|\tau|^{2}<\operatorname{Im} \tau^{1 / \alpha}$. Then the CR-function $f\left(z_{1}, z_{2}\right):=\left(z_{2}-c z_{1}^{1 / \alpha}\right)^{-1}$ on $V$ does not extend holomorphically to $W$. Since the above functions are uniformly approximated by holomorphic polynomials on compacta in $V$, it follows that the polynomial, rational and holomorphic hulls of $V$ (with respect to any Stein neighborhood) do not contain any wedge in $\mathbb{C}^{2}$ with edge $E$.

\section{Nonsmooth AnALYTiC Discs}

3.1. Basic definitions. The reader is referred to the books of Boggess [Bo91] and of BAOUEndi-Ebenfelt-Rothschild [BER99] for basic properties concerning analytic discs and the Hilbert transform. Here we survey some facts that will be used in this paper. We write $\Delta:=\{\tau \in \mathbb{C}:|\tau|<1\}$ for the unit disc and $\partial \Delta$ for its boundary. By an analytic disc in $\mathbb{C}^{n}$ we shall always mean a continuous map $A$ from the closure $\bar{\Delta}$ into $\mathbb{C}^{n}$ whose restriction to $\Delta$ is holomorphic. An analytic disc $A: \bar{\Delta} \rightarrow \mathbb{C}^{n}$ is said to be attached to a subset $S \subset \mathbb{C}^{n}$ if $A(\partial \Delta) \subset S$. (For attached analytic discs with merely measurable boundary values, see e.g. [Bo98], Bo99].)

Any analytic disc $A: \bar{\Delta} \rightarrow \mathbb{C}^{n}$ is uniquely determined by its boundary value $\left.A\right|_{\partial \Delta}$ and can be reconstructed by the unique harmonic extension of $\left.\operatorname{Re} A\right|_{\partial \Delta}$ and $\left.\operatorname{Im} A\right|_{\partial \Delta}$. Because of this uniqueness property we shall write the same letters for a disc and for its boundary value.

Vice versa, a given continuous complex function $A$ on $\partial \Delta$ is a boundary value of an analytic disc if and only if the real and imaginary parts of $A$ are related (up to an additive constant) by the Hilbert transform. Recall that the Hilbert transform of a real function $u$ on $\partial \Delta$ (in $L^{2}$ ) is the unique real function $v$ such that $v(0)=0$ and $u+i v$ extends holomorphically to $\Delta$ (in the sense of boundary values in $L^{2}$ ). Here we shall only consider functions in Hölder spaces $C^{\alpha}$ for $0<\alpha<1$ (see below for precise definitions) for which both Hilbert transform and harmonic extensions are also in $C^{\alpha}$ due to Privalov's classical theorem (see e.g. [Bo91]).

3.2. Preliminaries on Hölder spaces. In the sequel we shall write $a \lesssim b$ if there exists a universal constant $C>0$ such that the inequality $a \leq C b$ holds for all $a$ and $b$ under consideration. Let the unit circle $\partial \Delta$ be parameterized by $e^{i \theta}$, 
$\theta \in[0,2 \pi]$. If $f: \partial \Delta \rightarrow \mathbb{R}^{n}$ is a map, we write $f(\theta)$ instead of $f\left(e^{i \theta}\right)$ for brevity. By the $k$-th derivative $f^{(k)}$ of $f$ we mean the derivative with respect to $\theta$. For integers $k=0,1, \ldots$, we use the norm

$$
\|f\|_{k}:=\sum_{s=0}^{k} \sup _{\theta}\left|f^{(s)}(\theta)\right| .
$$

Further, we fix $0<\alpha<1$. Most of the following estimates hold also for $\alpha=1$. However, in order to have the boundedness of the Hilbert transform, we have to restrict to the case $0<\alpha<1$. We use the notation

$$
|f|_{\alpha}:=\sup _{\theta_{1} \neq \theta_{2}} \frac{\left|f\left(\theta_{1}\right)-f\left(\theta_{2}\right)\right|}{\left|\theta_{1}-\theta_{2}\right|^{\alpha}}, \quad\|f\|_{\alpha}:=\|f\|_{0}+|f|_{\alpha},
$$

and, for $k \geq 1$,

$$
\|f\|_{k, \alpha}:=\sum_{s=0}^{k}\left\|f^{(s)}\right\|_{\alpha} .
$$

The Hölder space $C^{\alpha}$ (resp. $\left.C^{k, \alpha}\right)$ is the space of all $C^{0}$ (resp. $C^{k}$ ) maps $f$ with $\|f\|_{\alpha}<\infty$ (resp. with $\left.\|f\|_{k, \alpha}<\infty\right)$.

If $f$ and $g$ are maps from $\partial \Delta$ into some matrix spaces such that the pointwise product $f g$ makes sense, we have

$$
\begin{aligned}
|f g|_{\alpha} & \leq \sup _{\theta_{1} \neq \theta_{2}} \frac{\left|f\left(\theta_{1}\right)\left(g\left(\theta_{1}\right)-g\left(\theta_{2}\right)\right)\right|}{\left|\theta_{1}-\theta_{2}\right|^{\alpha}}+\sup _{\theta_{1} \neq \theta_{2}} \frac{\left|\left(f\left(\theta_{1}\right)-f\left(\theta_{2}\right)\right) g\left(\theta_{2}\right)\right|}{\left|\theta_{1}-\theta_{2}\right|^{\alpha}} \\
& \leq\|f\|_{0}|g|_{\alpha}+|f|_{\alpha}\|g\|_{0},
\end{aligned}
$$

which implies the estimate

$$
\|f g\|_{\alpha} \leq\|f\|_{\alpha}\|g\|_{\alpha}
$$

showing that $C^{\alpha}$ is a Banach algebra. Analogously it follows that $C^{k, \alpha}$ is a Banach algebra for each $k$.

Now consider the case of compositions. Let $f: \partial \Delta \rightarrow \mathbb{R}^{n}$ be as before, and let $h: \mathbb{R}^{n} \rightarrow \mathbb{R}^{m}$ be a map of class $C^{1}$. We have

$$
\begin{aligned}
|h \circ f|_{\alpha} & =\sup _{\theta_{1} \neq \theta_{2}} \frac{\left|h\left(f\left(\theta_{1}\right)\right)-h\left(f\left(\theta_{2}\right)\right)\right|}{\left|\theta_{1}-\theta_{2}\right|^{\alpha}} \\
& \leq\left\|h^{\prime}\right\|_{0} \sup _{\theta_{1} \neq \theta_{2}} \frac{\left|f\left(\theta_{1}\right)-f\left(\theta_{2}\right)\right|}{\left|\theta_{1}-\theta_{2}\right|^{\alpha}} \leq\left\|h^{\prime}\right\|_{0}|f|_{\alpha},
\end{aligned}
$$

where $h^{\prime}$ is seen as a map from $\mathbb{R}^{n}$ into the vector space $\mathcal{L}\left(\mathbb{R}^{n}, \mathbb{R}^{m}\right)$ of all linear operators with the standard norm. From (4) we easily obtain

$$
\|h \circ f\|_{\alpha} \leq\|h\|_{0}+\left\|h^{\prime}\right\|_{0}|f|_{\alpha} .
$$


Furthermore, we will need an estimate for the difference of two compositions. Here we require $h$ to be of class $C^{2}$. Then, by (3), we obtain

$$
\begin{gathered}
\left\|h \circ f_{1}-h \circ f_{2}\right\|_{\alpha} \leq \int_{0}^{1}\left\|h^{\prime} \circ\left(t f_{1}+(1-t) f_{2}\right)\left[f_{1}-f_{2}\right]\right\|_{\alpha} d t \\
\leq \int_{0}^{1}\left\|h^{\prime} \circ\left(t f_{1}+(1-t) f_{2}\right)\right\|_{\alpha}\left\|f_{1}-f_{2}\right\|_{\alpha} d t \\
\leq \int_{0}^{1}\left(\left\|h^{\prime}\right\|_{0}+\left\|h^{\prime \prime}\right\|_{0}\left\|t f_{1}+(1-t) f_{2}\right\|_{\alpha}\right)\left\|f_{1}-f_{2}\right\|_{\alpha} d t \\
\leq\left(\left\|h^{\prime}\right\|_{0}+\frac{1}{2}\left\|h^{\prime \prime}\right\|_{0}\left(\left\|f_{1}\right\|_{\alpha}+\left\|f_{2}\right\|_{\alpha}\right)\right)\left\|f_{1}-f_{2}\right\|_{\alpha} .
\end{gathered}
$$

3.3. Function spaces with singularities. In this section we introduce Banach spaces of functions on $\partial \Delta$ and on $\bar{\Delta}$ that are (at least) $C^{1}$ everywhere outside the point $1 \in \partial \Delta$ and have a prescribed singularity at $\tau=1$. These spaces will play a crucial role in the construction of nonsmooth attached analytic discs in this paper.

In the sequel, for $0<\alpha<1$, we always consider the principle branch of $(1-\tau)^{\alpha}$ on $\bar{\Delta}$ which is real and positive on the segment $[-1,1]$. For each $\alpha$ denote by $d=d(\alpha)$ the unique positive integer such that $d \alpha<1 \leq(d+1) \alpha$ Then $d$ is the maximal power such that $(1-\tau)^{d \alpha} \notin C^{1, \beta}$ for any $0<\beta<1$. For the sequel we fix any $\beta$ satisfying

$$
\begin{cases}0<\beta \leq(d+1) \alpha-1 & \text { if }(d+1) \alpha>1, \\ 0<\beta \leq(d+2) \alpha-1 & \text { if }(d+1) \alpha=1\end{cases}
$$

(we always have $(d+1) \alpha-1<1$ ). Then we have $\beta<\alpha$ and $(1-\tau)^{s \alpha} \in C^{1, \beta}$ if and only if $s=0$ or $s>d$. Motivated by these considerations, we introduce function spaces spanned by all powers of $(1-\tau)^{\alpha}$ and the space $C^{1, \beta}$ as follows.

Denote by $\mathbb{C}_{d}\left[(1-\tau)^{\alpha}\right]$ and $\mathbb{C}_{d}\left[(1-\tau)^{\alpha},(1-\bar{\tau})^{\alpha}\right]$ the (finite-dimensional) spaces of complex polynomials of degree at most $d$ in the corresponding variables. By a slight abuse we use the same notation for the spaces of restrictions of the polynomials to $\partial \Delta$ and to $\bar{\Delta}$ respectively. In order to exclude constant functions from $C^{1, \beta}$, we consider the subspace of all functions $f \in C^{1, \beta}$ with $f(1)=0$ denoted by $C_{1}^{1, \beta}$.

We now define

$$
\begin{aligned}
& \mathcal{P}^{\alpha}(\partial \Delta):=\mathbb{C}_{d}\left[(1-\tau)^{\alpha}\right]+C_{1}^{1, \beta}(\partial \Delta) \subset C^{0}(\partial \Delta), \\
& \mathcal{P}^{\alpha}(\bar{\Delta}):=\mathbb{C}_{d}\left[(1-\tau)^{\alpha},(1-\bar{\tau})^{\alpha}\right]+C_{1}^{1, \beta}(\bar{\Delta}) \subset C^{0}(\bar{\Delta}) .
\end{aligned}
$$

Our choice of the spaces is motivated by the following basic property:

Lemma 3.1. Both sums in (8) are direct, i.e. any function $f \in \mathcal{P}^{\alpha}(\partial \Delta)$ (resp. $f \in \mathcal{P}^{\alpha}(\bar{\Delta})$ ) is uniquely decomposed as a sum $f=p+g$ with $g \in C_{1}^{1, \beta}(\partial \Delta)$ (resp. $g \in C_{1}^{1, \beta}(\bar{\Delta})$ ) and $p$ a polynomial in the corresponding space.

Proof. We show that the decomposition $f=p+g$ is uniquely determined by the asymptotics of $f$ at $1 \in \partial \Delta$. For $\tau=e^{i \theta} \in \partial \Delta$ and any $s$, we have

$$
(1-\tau)^{s \alpha}=(1-\cos \theta-i \sin \theta)^{s \alpha}=(-i \theta)^{s \alpha}\left(1+\theta r_{s}(\theta)\right)
$$

with $r_{s}(\theta)$ real-analytic for $\theta \in[-\pi, \pi]$, where we used the standard power series expansions of $\sin \theta$ and $\cos \theta$ at $\theta=0$. Hence, for any two decompositions $f=$ $p_{1}+g_{1}=p_{2}+g_{2}$ as in the lemma, we must have $p_{1}\left((-i \theta)^{\alpha}\right)-p_{2}\left((-i \theta)^{\alpha}\right) \in C_{1}^{1, \beta}$, 
which is only possible for $p_{1}=p_{2}$ and therefore $g_{1}=g_{2}$. Similarly, the uniqueness of the decomposition in $\mathcal{P}^{\alpha}(\bar{\Delta})$ follows from the asymptotics of the powers $(1-\tau)^{s \alpha}$ at $\tau=1$ for $\tau \in \bar{\Delta}$.

We define the norm on each polynomial space to be the sum of the absolute values of the coefficients. We also consider the standard norm $\|\cdot\|_{1, \beta}$ on $C_{1}^{1, \beta}$. Then the uniqueness of the decomposition allows us to define the norm $\|f\|_{(\alpha)}$ of an element $f=p+g$ of $\mathcal{P}^{\alpha}(\partial \Delta)$ (resp. of $\mathcal{P}^{\alpha}(\bar{\Delta})$ ) with $p$ and $g$ as in Lemma 3.1 to be the sum of the norms of $p$ and $g$.

Lemma 3.2. There exists a constant $C>0$ such that the spaces $\mathcal{P}^{\alpha}(\partial \Delta)$ and $\mathcal{P}^{\alpha}(\bar{\Delta})$ with the norm $C\|\cdot\|_{(\alpha)}$ become Banach algebras.

Proof. We consider the space $\mathcal{P}^{\alpha}(\partial \Delta)$; the proof for the space $\mathcal{P}^{\alpha}(\bar{\Delta})$ is completely analogous. The only nontrivial statement is the behavior with respect to the multiplication. The property

$$
C\|f g\|_{(\alpha)} \leq C^{2}\|f\|_{(\alpha)}\|g\|_{(\alpha)}
$$

can be easily checked if both $f$ and $g$ are either polynomials or in $C_{1}^{1, \beta}$. It remains to consider the case when $f(\tau)=(1-\tau)^{s \alpha}$ and $g \in C_{1}^{1, \beta}$. By removing the linear terms, we may assume that $g(1)=g^{\prime}(1)=0$ and hence $|g(\tau)| \leq\|g\|_{1, \beta}|1-\tau|$, $\left|g^{\prime}(\tau)\right| \leq\|g\|_{1, \beta}|1-\tau|^{\beta}$. We have

$$
(f g)^{\prime}(\tau)=s \alpha(1-\tau)^{s \alpha-1} g(\tau)+(1-\tau)^{s \alpha} g^{\prime}(\tau) .
$$

Since $s \alpha \geq \alpha>\beta$, the second term is a product of functions in $C^{\beta}$, hence it is in $C^{\beta}$ with its norm estimated by $\|f\|_{(\alpha)}\|g\|_{(\alpha)}$. To show that the first term is also in $C^{\beta}$, we estimate its derivative

$$
\left|\left(s \alpha(1-\tau)^{s \alpha-1} g(\tau)\right)^{\prime}\right| \lesssim|1-\tau|^{\beta-2}|g(\tau)|+|1-\tau|^{s \alpha-1}\left|g^{\prime}(\tau)\right| \lesssim\|g\|_{1, \beta}|1-\tau|^{\beta-1}
$$

which implies, by integration, that also the first term on the right-hand side of (10) is in $C^{\beta}$ with its norm estimated by $\|g\|_{1, \beta}$. Summarizing, we have $f g \in C_{1}^{1, \beta}(\partial \Delta)$ with $\|f g\|_{(\alpha)} \lesssim\|f\|_{(\alpha)}\|g\|_{(\alpha)}$. Then the estimate (9) is easily obtained by choosing a suitable constant $C$.

For the sequel we rescale the norm according to Lemma 3.2 to obtain the inequality $\|f g\|_{(\alpha)} \leq\|f\|_{(\alpha)}\|g\|_{(\alpha)}$ for all $f, g \in \mathcal{P}^{\alpha}(\partial \Delta)$ (resp. $\left.f, g \in \mathcal{P}^{\alpha}(\bar{\Delta})\right)$ without any constant $C$.

3.4. Boundedness of the Hilbert transform. In the following, when we write $\mathcal{P}^{\alpha}(K)$, we always mean that $K$ is either $\partial \Delta$ or $\bar{\Delta}$. We define spaces analogous to $\mathcal{P}^{\alpha}(K)$ for $\mathbb{R}^{n}$-valued functions by using the standard embedding $\mathbb{R}^{n} \subset \mathbb{C}^{n}$ and by defining a function $f: K \rightarrow \mathbb{R}^{n}$ to be in $\mathcal{P}^{\alpha}\left(K, \mathbb{R}^{n}\right)$ if and only if each component of $f$ is in the corresponding function space $\mathcal{P}^{\alpha}(K)$. Thus a function is in $\mathcal{P}^{\alpha}\left(K, \mathbb{C}^{n}\right)$ if and only if its real and imaginary part are both in $\mathcal{P}^{\alpha}\left(K, \mathbb{R}^{n}\right)$. With the norm being the sum of norms of components, we obtain a natural Banach space structure on $\mathcal{P}^{\alpha}\left(K, \mathbb{R}^{n}\right)$. The following basic property follows directly from the construction and from Privalov's theorem:

Lemma 3.3. The Hilbert transform is a bounded linear operator on $\mathcal{P}^{\alpha}(\partial \Delta)$. 
3.5. Composition with smooth functions. As before, for $h: \mathbb{R}^{n} \rightarrow \mathbb{R}^{m}$, we view the derivative $h^{\prime}$ as a map from $\mathbb{R}^{n}$ into $\mathcal{L}\left(\mathbb{R}^{n}, \mathbb{R}^{m}\right)$ and enclose the vector argument in brackets [.] to avoid confusion with other arguments.

Let $d \geq 1$ and $0<\beta<\alpha$ be chosen as in the previous section. We begin with a special case of composition, where the map $h$ vanishes of order higher than $d$ at 0 . We then show that the composition $h \circ f$ is always in $C^{1, \beta}$ for $f \in \mathcal{P}^{\alpha}(K)$ with $f(1)=0$.

Lemma 3.4. For $f \in \mathcal{P}^{\alpha}\left(K, \mathbb{R}^{n}\right)$ with $f(1)=0$ and $h \in C^{d+2}\left(\mathbb{R}^{n}, \mathbb{R}^{m}\right)$ with vanishing derivatives $h^{(k)}(0), 1 \leq k \leq d$, the composition $h \circ f$ is always in $C^{1, \beta}\left(K, \mathbb{R}^{m}\right)$ and we have the estimate

$$
\|h \circ f\|_{1, \beta} \lesssim\left(1+\|f\|_{(\alpha)}^{d+1}\right)\|h\|_{d+2} .
$$

Proof. We write $f=p+g$ as in Lemma 3.1 and compute the derivative

$$
(h(f))^{\prime}(\tau)=h^{\prime}(f(\tau))\left[p^{\prime}(\tau)\right]+h^{\prime}(f(\tau))\left[g^{\prime}(\tau)\right] .
$$

Since $\beta<\alpha$ and $f \in C^{\alpha}$, we always have $f \in C^{\beta}$ with $\|f\|_{\beta} \lesssim\|f\|_{(\alpha)}$, and therefore $h^{\prime} \circ f \in C^{\beta}$ with $\left\|h^{\prime} \circ f\right\|_{\beta} \lesssim\left(1+\|f\|_{(\alpha)}\right)\|h\|_{2}$ by (5). Since $g^{\prime} \in C^{\beta}$ and $C^{\beta}$ is a Banach algebra, the second term on the right-hand side of (12) is in $C^{\beta}$ and its norm is estimated by the right-hand side of (11).

It remains to show the same estimate for the first term on the right-hand side of (12). Since $(1-\tau)^{\alpha} \in C^{\beta}$, multiplication by $(1-\tau)^{\alpha}$ or $(1-\bar{\tau})^{\alpha}$ preserves the class $C^{\beta}$. Hence it suffices to prove the estimate

$$
\left\|h^{\prime}(f(\tau))\left[p^{\prime}(\tau)\right]\right\|_{\beta} \lesssim\left(1+\|f\|_{(\alpha)}^{d+1}\right)\|h\|_{d+2}
$$

for $p(\tau)=(1-\tau)^{\alpha}$ (the case $p(\tau)=(1-\bar{\tau})^{\alpha}$ is completely analogous). To show (13) we compute the derivative:

$$
\left(h^{\prime}(f(\tau))\left[(1-\tau)^{\alpha-1}\right]\right)^{\prime}(\tau)=h^{\prime \prime}(f(\tau))\left[f^{\prime}(\tau)\right]\left[(1-\tau)^{\alpha-1}\right]+(\alpha-1) h^{\prime}(f(\tau))\left[(1-\tau)^{\alpha-2}\right] .
$$

We now use the vanishing of the derivatives of $h$ at 0 . Without loss of generality, $h(0)=0$. By the assumptions, we have

$$
|h(x)| \lesssim\|h\|_{d+2}|x|^{d+2}, \quad\left|h^{\prime}(x)\right| \lesssim\|h\|_{d+2}|x|^{d+1}, \quad\left|h^{\prime \prime}(x)\right| \lesssim\|h\|_{d+2}|x|^{d} .
$$

We use (15) together with

$$
|f(\tau)| \lesssim\|f\|_{(\alpha)}|1-\tau|^{\alpha} \text { and }\left|f^{\prime}(\tau)\right| \lesssim\|f\|_{(\alpha)}|1-\tau|^{\alpha-1}
$$

to estimate the first term on the right-hand side of (14):

$$
\left|h^{\prime \prime}(f(\tau))\left[f^{\prime}(\tau)\right]\left[(1-\tau)^{\alpha-1}\right]\right| \lesssim\|f\|_{(\alpha)}^{d+1}\|h\|_{d+2}|1-\tau|^{(d+2) \alpha-2} .
$$

Similarly we estimate the second term on the right-hand side of (14):

$$
\left|h^{\prime}(f(\tau))\left[(1-\tau)^{\alpha-2}\right]\right| \lesssim\|f\|_{(\alpha)}^{d+1}\|h\|_{d+2}|1-\tau|^{(d+2) \alpha-2} .
$$

The required estimate (13) is obtained by integrating (16) and (17).

Remark 3.5. The smoothness $h \in C^{d+2}$ is only needed in the case $(d+1) \alpha=1$; otherwise the proof gives the same estimates with $d+2$ replaced by $d+1$. In particular, for $d=1$, i.e. $1 / 2<\alpha<1$, the proof goes through for $h \in C^{d+1}=C^{2}$. For uniformity we write $d^{\prime}=d^{\prime}(\alpha)=d$ for $(d+1) \alpha>1$, and $d^{\prime}=d+1$ otherwise (thus $d^{\prime}=1$ if and only if $1 / 2<\alpha<1$ ). Then the conclusion of Lemma 3.4 holds for $d+2$ replaced replaced by $d^{\prime}+1$. 
The behavior of the spaces $\mathcal{P}^{a}$ with respect to the composition with general smooth functions (not only vanishing up to a certain order) is expressed in the following lemma.

Lemma 3.6. For $f \in \mathcal{P}^{\alpha}\left(K, \mathbb{R}^{n}\right)$ and $h \in C^{d^{\prime}+1}\left(\mathbb{R}^{n}, \mathbb{R}^{m}\right)$, the composition $h \circ f$ is always in $\mathcal{P}^{\alpha}\left(K, \mathbb{R}^{m}\right)$ and we have the estimate

$$
\|h \circ f\|_{(\alpha)} \lesssim\left(1+\|f\|_{(\alpha)}^{d+1}\right)\|h\|_{d^{\prime}+1} .
$$

Proof. By a coordinate translation in $\mathbb{R}^{n}$, we may assume $f(1)=0$; in particular,

$$
|f(\tau)| \lesssim\|f\|_{(\alpha)}|1-\tau|^{\alpha} .
$$

In the case when $h$ is a polynomial of degree at most $d$, the statement of the lemma follows from Lemma 3.2. In general, $h$ can be written as the sum of its Taylor polynomial of order $d$ at 0 and a function satisfying the vanishing assumption of Lemma 3.4 and the required conclusion is obtained by combining Lemma 3.4 with the above remark.

We now use a result of [L00] to conclude the differentiability of the composition operator acting on $\mathcal{P}^{\alpha}\left(K, \mathbb{R}^{n}\right)$ :

Proposition 3.7. For $k \geq 1$, the composition $(h, f) \mapsto h \circ f$ defines a $C^{k}$ map $c: C^{k+d^{\prime}+1}\left(\mathbb{R}^{n}, \mathbb{R}^{m}\right) \times \mathcal{P}^{\alpha}\left(K, \mathbb{R}^{n}\right) \rightarrow \mathcal{P}^{\alpha}\left(K, \mathbb{R}^{m}\right)$ whose first derivative is given by

$$
c^{\prime}(h, f)[\dot{h}, \dot{f}](\theta)=\dot{h}(f(\theta))+h^{\prime}(f(\theta))[\dot{f}(\theta)] .
$$

Proof. We show that the statement is a special case of Theorems 3.1 in [L00]. It is sufficient to consider the maps $f$ with values in a given $C^{\infty}$ smoothly bounded domain $\Omega \subset \mathbb{R}^{n}$ and the maps $h$ defined in $\Omega$. We adapt the notation of [L00] to our situation. As in [L00], denote by $\mathcal{P}\left(\mathbb{R}^{n}\right)$ the space of polynomials, and define $\mathcal{Y}:=C^{d^{\prime}+1}(\bar{\Omega})$ and the norm $\|p\|_{\mathcal{Y}}$ of $p \in \mathcal{P}\left(\mathbb{R}^{n}\right)$ to be the $C^{d^{\prime}+1}$ norm of $\left.p\right|_{\Omega}$. The norm $\|\cdot\| \mathcal{Y}$ induces the sequence of norms $\|p\|_{\mathcal{Y}_{r}}:=\sum_{s=0}^{r}\left\|p^{(s)}\right\| \mathcal{Y}$ for every $r \geq 1$, where $\mathcal{Y}_{r}:=C^{r+d^{\prime}+1}(\bar{\Omega})$ is the completion of $\mathcal{P}\left(\mathbb{R}^{n}\right)$ with respect to $\|\cdot\|_{\mathcal{Y}_{r}}$.

We now apply Theorems 3.1 and 4.1 in [L00] to the case where $\mathcal{X}=\widetilde{\mathcal{X}}:=\mathcal{P}^{\alpha}(K)$, $\mathcal{J}:=$ id and $\mathcal{A}$ is the set of all $\mathcal{F}^{\alpha}$ maps with values in $\Omega$. Then the estimate (3.1) in [L00] follows from Lemma 3.6] Furthermore, Lemma 3.6] implies that the map $h \mapsto h \circ f$ is continuous from $\mathcal{Y}$ to $\widetilde{\mathcal{X}}$ for fixed $f$. Hence the composition map $\widetilde{\mathcal{A}}(h, f):=h \circ f$ satisfies both properties (i) and (ii) in Theorem 3.1 and therefore coincides with the abstract composition operator constructed there. Then the required conclusion follows from Theorem 4.1 in [L00] (with "*" being the multiplication in $\left.\mathcal{P}^{\alpha}(K)\right)$.

\section{Attaching Nonsmooth AnALYtic Discs to GeneriC Manifolds}

4.1. Analytic discs in $\mathcal{P}^{\alpha}$. Let $M \subset \mathbb{C}^{n}$ be a generic submanifold of class $C^{k+d^{\prime}+1}$ $(k \geq 1)$ through 0 that is locally represented as a graph

$$
M=\left\{(x+i y, w) \in \mathbb{C}^{n-m} \times \mathbb{C}^{m}: y=h(x, w)\right\}
$$

with $h(0)=0, h^{\prime}(0)=0$. Since $\mathcal{P}^{\alpha} \subset C^{\alpha}$, the classical statements about small analytic $\operatorname{discs} A(\cdot)=(z(\cdot), w(\cdot))$ attached to $M$ ([Bi65], [HT78], T88], [Bo91], [BER99]) yield existence (and uniqueness) of such discs in $C^{\alpha}$ with a prescribed " $w$-component" $w(\cdot) \in C^{\alpha}$. We wish to show that, if the " $w$-component" is in $\mathcal{P}^{\alpha}$, the whole disc is actually in $\mathcal{P}^{\alpha}$. Furthermore, it will be also important for 
our purposes to know that the analytic discs in $\mathcal{P}^{\alpha}$ smoothly depend on all their parameters. It is known that regularity properties of the parameter dependence of attached analytic discs may differ from regularity properties of each single disc (see e.g. [T93]).

We obtain regularity for both single discs and their parameter dependence as a consequence of Proposition 3.7 and the implicit function theorem in the Banach space $\mathcal{P}^{\alpha}$. We choose $d^{\prime}$ as in Remark 3.5 .

Proposition 4.1. Let $h=h_{0}$ be of class $C^{d^{\prime}+2}$, and let $M=M_{0}$ be given by (21). Then, for every sufficiently small $x \in \mathbb{R}^{n-m}, w(\cdot) \in \mathcal{P}^{\alpha}$, where $w(\cdot)$ is holomorphically extendible to $\Delta$, and for every $h$ sufficiently close to $h_{0}$ in $C^{d^{\prime}+2}$, there exists a unique sufficiently small analytic disc $A(\cdot)=(z(\cdot), w(\cdot))$ in $\mathcal{P}^{\alpha}$ attached to $M$ such that $\operatorname{Re} z(1)=x$. For $h \in C^{k+d^{\prime}+1}(k \geq 1)$, the disc $A \in \mathcal{P}^{\alpha}$ depends in a $C^{k}$ fashion on the parameters $x \in \mathbb{R}^{n-m}, w \in \overline{\mathcal{P}}^{\alpha}$ and $h \in C^{k+d^{\prime}+1}$.

Proof. By a complex-linear change of the coordinate $z \in \mathbb{C}^{n-m}$ (without changing $w)$ we may assume that $h_{0}^{\prime}(0)=0$. The required analytic disc $A(\cdot)=(x(\cdot)+$ $i y(\cdot), w(\cdot))$ is obtained by solving the Bishop's equation

$$
x(\cdot)+T_{1} h(x(\theta), w(\theta))(\cdot)=x_{0}
$$

and putting $y(\cdot)=h(x(\cdot), w(\cdot))$. Here $T_{1}$ denotes the modified Hilbert transform (introduced by BoGgEss-PITTS [BP85]) such that $y(\cdot)+i T_{1} y(\cdot)$ extends holomorphically to $\Delta$ and $T_{1} y(1)=0$. Since the Hilbert transform is bounded in $\mathcal{P}^{\alpha}$ by Lemma 3.3 and the composition $h \circ(x, w)$ is smooth in its components by Proposition 3.7, the implicit function theorem yields the solvability of (22) and the required smooth dependence of the solution on all parameters.

4.2. Infinitesimal deformations of attached $\mathcal{P}^{\alpha}$ discs. The deformation theory for smooth attached analytic discs due to Tumanov [T88] and BAOUEndRothSCHILD-TRÉPREAU [BRT94] is based on the notion of the so-called "defect" of an analytic disc. In this paper we shall use a construction that is independent of a defect and works equally well for all discs. It is closely related to the construction of "partial lifts" due to BARACCO and the second author [BZ01]. As before, consider coordinates $(x+i y, w) \in \mathbb{C}^{n-m} \times \mathbb{C}^{m}$, where a submanifold $M \subset \mathbb{C}^{n}$ is given by $y=h(x, w)$ with $h \in C^{\kappa}$. We identify the cotangent bundle $T^{*} \mathbb{C}^{n}$ with the space of all $(1,0)$ covectors. The conormal bundle $T_{M}^{*} \mathbb{C}^{n}$ of $M$ in $\mathbb{C}^{n}$ is the set of all covectors in $\left.T^{*} \mathbb{C}^{n}\right|_{M}$ whose restrictions to $T M$ are purely imaginary. Then $T_{M}^{*} \mathbb{C}^{n}$ is a real $C^{\kappa-1}$ submanifold of $T^{*} \mathbb{C}^{n}$ that is not necessarily $\mathrm{CR}$ in general. However, the restriction map naturally identifies $T_{M}^{*} \mathbb{C}^{n}$ with a generic submanifold of $T^{*} \mathbb{C}^{n-m}$ (where $\mathbb{C}^{n-m} \cong \mathbb{C}_{x+i y}^{n-m} \times\{0\}$ ) as follows. Denote by $\pi: T^{*} \mathbb{C}^{n} \rightarrow \mathbb{C}^{n}$ the natural projection and by $\left(T_{M}^{*} \mathbb{C}^{n}\right)_{p}$ the fiber over a point $p \in M$. By the construction, each fiber $\left(T_{M}^{*} \mathbb{C}^{n}\right)_{p}$ is naturally identified with a maximal totally real linear subspace of $T_{p}^{*} \mathbb{C}^{n-m}$. Denote by $\mathrm{GL}\left(\mathbb{C}^{n-m}\right)$ the group of all linear automorphisms of $\mathbb{C}^{n-m}$. Then, given $p \in M$, the set $\mathcal{G}^{*}(p)$ of all $\left(q, G^{*}\right) \in M \times \mathrm{GL}\left(\mathbb{C}^{n-m}\right)$ with $G^{*}\left(\left(T_{M}^{*} \mathbb{C}^{n}\right)_{p}\right)=\left(T_{M}^{*} \mathbb{C}^{n}\right)_{q}$ is a generic submanifold in $\mathbb{C}^{n} \times \mathrm{GL}\left(\mathbb{C}^{n-m}\right)$ with maximal totally real fibers over $M$. The following lemma is a consequence of Proposition 4.1 applied for each $p=A(1)$ to the generic submanifold $\mathcal{G}^{*}(p) \subset \mathbb{C}^{n} \times \mathrm{GL}\left(\mathbb{C}^{n-m}\right)$. 
Lemma 4.2. Let $A$ be a sufficiently small analytic disc in $\mathbb{C}^{n}$ of class $\mathcal{P}^{\alpha}$ attached to a submanifold $M \subset \mathbb{C}^{n}$ of class $C^{d^{\prime}+3}$. Then there exists a unique $\operatorname{GL}\left(\mathbb{C}^{n-m}\right)$ valued analytic disc $G^{*}$ of class $\mathcal{P}^{\alpha}$ such that $G^{*}(1)=\mathrm{id}$ and, for $\theta \in \partial \Delta$,

$$
G^{*}(\theta)\left(\left(T_{M}^{*} \mathbb{C}^{n}\right)_{A(1)}\right)=\left(T_{M}^{*} \mathbb{C}^{n}\right)_{A(\theta)} .
$$

Remark 4.3. The statement or Lemma 4.2 clearly holds also in the classical setting, where the discs are considered of class $C^{k, \alpha}$.

Lemma 4.2 establishes existence of a "connection" $G^{*}$ on $T_{M}^{*} \mathbb{C}^{n}$ over $\partial \Delta$ that defines, for each $\theta_{1}, \theta_{2} \in \partial \Delta$, a linear isomorphism between the fibers $\left(T_{M}^{*} \mathbb{C}^{n}\right)_{A\left(\theta_{1}\right)}$ and $\left(T_{M}^{*} \mathbb{C}^{n}\right)_{A\left(\theta_{2}\right)}$. (In general, this connection depends on the choice of the splitting $\mathbb{C}^{n}=\mathbb{C}^{n-m} \times \mathbb{C}^{m}$.) By duality, $G^{*}$ yields an isomorphism between $\left(T_{M} \mathbb{C}^{n}\right)_{A\left(\theta_{1}\right)}$ and $\left(T_{M} \mathbb{C}^{n}\right)_{A\left(\theta_{2}\right)}$, where $T_{M} \mathbb{C}^{n}$ stands for the quotient $\left(\left.T \mathbb{C}^{n}\right|_{M}\right) / T M$. We write $G$ for the corresponding GL $\left(\mathbb{C}^{n-m}\right)$-valued analytic disc that yields the dual connection on $T_{M} \mathbb{C}^{n}$ over $A$. We note that, in general, this connection may depend on the coordinates (essentially on the choice of the coordinate subspace $\mathbb{C}^{n-m} \subset \mathbb{C}^{n}$ ), in contrast to the connection defined in [T94], which does not depend on the coordinates but, on the other hand, is only defined in a quotient bundle of $T_{M} \mathbb{C}^{n}$. However, in order to obtain propagation of all directions normal to $M$ we need a connection on the bundle $T_{M} \mathbb{C}^{n}$ itself.

4.3. Radial derivatives of attached analytic discs in $\mathcal{P}^{\alpha}$. In the following we assume $\alpha>1 / 2$ and $0<\beta \leq 2 \alpha-1, d^{\prime}=1$.

Lemma 4.4. Let $A$ be an analytic $\mathcal{P}^{\alpha}$ disc attached to a generic submanifold $M \subset \mathbb{C}^{n}$ of class $C^{2}$, and set $p:=A(1) \in M$. Suppose that $A$ is sufficiently small in $\mathcal{P}^{\alpha}$. Then (in the given coordinates) there is a unique representation

$$
A(\tau)=p+(1-\tau)^{\alpha} A_{0}+A_{1}(\tau)
$$

with $A_{0} \in T_{p}^{c} M, A_{1}(\cdot) \in C^{1, \beta}$ and $A_{1}(1)=0$.

Proof. Without loss of generality, $p=0$ and $M$ is generic and given by (21) with $h(0)=0, h^{\prime}(0)=0$. Since $A$ is attached to $M$, we have $y(\tau)=h(x(\tau), w(\tau))$ for $\tau \in \partial \Delta$ in the notation of (21). Then it follows from Lemma 3.4 (see also Remark 3.5) that $y(\cdot) \in C^{1, \beta}(\partial \Delta)$. Hence the Hilbert transform $x(\cdot)$ is also in $C^{1, \beta}(\partial \Delta)$, and therefore the holomorphic extension $z(\cdot)=x(\cdot)+i y(\cdot)$ is in $C^{1, \beta}(\bar{\Delta})$. The existence of the representation now follows from the construction of the space $\mathcal{P}^{\alpha}$. The uniqueness is clear, since it generally holds for maps of class $\mathcal{P}^{\alpha}$ by Lemma 3.1

In the sequel we shall write $\partial_{r}$ for the radial derivative in $\Delta$. Denote by $[v] \epsilon$ $T_{M} \mathbb{C}^{n}$ the equivalence class defined by a tangent vector $v$. Since by Lemma 4.4 the normal component of $A$ is $C^{1, \beta}$, it makes sense to write $\left[\partial_{r} A(1)\right] \in\left(T_{M} \mathbb{C}^{n}\right)_{A(1)}$, even though $\partial_{r} A(1)$ may not exist (in fact, it will never exist in our case). The following statement shows that, if $A$ is tangent, $G(\theta)$ describes the direction of the deformation of $A$.

Proposition 4.5. Suppose that $A$ is tangent to $M$ at $p:=A(1)$, i.e. $\left[\partial_{r} A(1)\right]=0$. Let $M^{\prime} \subset \mathbb{C}^{n}$ be a $C^{k}$-smooth $(k \geq 4)$ submanifold with boundary $M$ at a point $q \in A(\partial \Delta)$ with $C_{q} M^{\prime}=T_{q} M \oplus \mathbb{R}_{+} v$ for suitable $v \in T_{q} \mathbb{C}^{n}$. Then, for any $\varepsilon>0$, there exists a $C^{k}$-smooth family of submanifolds $M_{\eta} \subset M \cup M^{\prime}, 0 \leq \eta \leq \eta_{0}$, with 
$M_{0}=M$ such that the (unique) analytic disc $A_{\eta}(\cdot)=\left(z_{\eta}(\cdot), w(\cdot)\right)$ attached to $M_{\eta}$ with $A_{\eta}(1)=p$ and with fixed " $w$-component" $w(\cdot)$ has the property

$$
\left[\partial_{r} A_{\eta}(1)\right]=\eta\left(G\left(\tau_{0}\right)^{-1}[v]+\left[v_{0}\right]\right)+o(\eta), \quad \eta \rightarrow 0,
$$

for some vector $v_{0} \in T_{p} \mathbb{C}^{n}$ with $\left|v_{0}\right|<\varepsilon$.

We obtain Proposition 4.5 as an immediate consequence of the following more general statement:

Proposition 4.6. In the setting of Proposition 4.5 let us drop the assumption that $A$ is tangent. Then we have the same conclusion, with (24) being replaced by

$$
\left[\partial_{r} A_{\eta}(1)\right]=\left[\partial_{r} A(1)\right]+\eta\left(G\left(\tau_{0}\right)^{-1}[v]+\left[v_{0}\right]\right)+o(\eta), \quad \eta \rightarrow 0,
$$

for some vector $v_{0} \in T_{p} \mathbb{C}^{n}$ with $\left|v_{0}\right|<\varepsilon$.

Proof. To construct the required deformation $M_{\eta}, 0 \leq \eta \leq \eta_{0}$, consider real coordinates $x \in \mathbb{C}^{n} \cong \mathbb{R}^{2 n}$ vanishing at $q$, where $M$ is given by $x_{1}=\cdots=x_{s}=0$ and $M^{\prime}$ by $x_{1}=\cdots=x_{s-1}=0, x_{s} \geq 0$. Let $\varphi$ be a nonnegative function with compact support in a sufficiently small neighborhood of $q$ in $M$. Then define $M_{\eta}$ to be given by $x_{s}=\eta \varphi\left(x_{s+1}, \ldots, x_{2 n}\right)$ near $q$ and to coincide with $M$ outside the support of $\varphi$.

Let $A_{\eta}$ be the analytic disc attached to $M_{\eta}$ with $A_{\eta}(1)=p$ and with the same $w$-component as $A$. By Proposition 4.1, the derivative $\dot{A}$ of $A_{\eta}$ with respect to $\eta$ for $\eta=0$ exists and belongs to $\mathcal{P}^{\alpha}$. Since only the "z-component" of $A_{\eta}$ changes, $\dot{A}=(\dot{z}, 0)$. Let $G^{*}$ be given by Lemma 4.2 Then, for any covector $\xi \in\left(T_{M}^{*} \mathbb{C}^{n}\right)_{p}$, $\psi(\tau):=\left(G^{*}(\tau) \xi\right)[\dot{A}(\tau)]$ is a holomorphic function in $\Delta$. By the construction, the real part of $G^{*}(\tau) \xi$ vanishes on $\partial \Delta$. Hence $\operatorname{Re} \psi$ vanishes on $\partial \Delta$ away from the support of $\varphi$, where $M$ is not deformed.

We now choose $\xi$ such that

$$
\operatorname{Re}\left(G^{*}\left(\tau_{0}\right) \xi\right)[v] \geq \delta|\xi|
$$

for a fixed small $\delta>0$. If the deformation of $M$ takes place only in a sufficiently small neighborhood of $q$, then the direction of $[\dot{A}(\tau)]$ differs only slightly from $[v]$ for $\tau$ near $\tau_{0}$, and $[\dot{A}(\tau)]=0$ for $\tau$ far from $\tau_{0}$. Hence $\operatorname{Re} \psi(1)=0$ and (26) implies $\operatorname{Re} \psi(\tau) \geq 0$ everywhere for $\tau \in \partial \Delta$. Since $\operatorname{Re} \psi$ is harmonic in $\Delta$, by the Hopf lemma, the radial derivative $\operatorname{Re} \xi\left[\partial_{r} \dot{A}(1)\right]$ is positive. Since $\xi$ can be any covector satisfying (26), it follows that

$$
\left|\left[\partial_{r} \dot{A}(1)\right]-\lambda G\left(\tau_{0}\right)^{-1}[v]\right|<\varepsilon
$$

for some $\lambda>0$ and sufficiently small $\delta>0$ (depending on $\varepsilon$ ). By a linear change of the parameter $\eta$ we can achieve $\lambda=1$. Then, to show (25), it remains to observe that the radial derivative $\left[\partial_{r} A_{\eta}(1)\right]$ is continuously differentiable in $\eta$ with $\left.\frac{\partial}{\partial \eta}\left[\partial_{r} A_{\eta}(1)\right]\right|_{\eta=0}=\left[\partial_{r} \dot{A}(1)\right]$.

As an immediate application of Proposition 4.6 we obtain:

Corollary 4.7. Let $M \subset \mathbb{C}^{n}$ be a generic $C^{k}$-smooth $(k \geq 4)$ submanifold through $p=0$, and let $A$ be a sufficiently small analytic disc of class $\mathcal{P}^{\alpha}$ attached to $M$. Then, if $M_{1}^{\prime}, \ldots, M_{s}^{\prime}$ are $C^{k}$-smooth submanifolds with boundary $M$ at a point $q \in$ $A(\partial \Delta)$ in s linearly independent directions $\left[v_{1}\right], \ldots,\left[v_{s}\right] \in\left(T_{M} \mathbb{C}^{n}\right)_{q}$, then there exist s submanifolds $M_{j} \subset M \cup M_{j}^{\prime}$ of class $C^{k}$, of the same dimension as $M$ and arbitrarily close to $M$ in the $C^{k}$ norm, such that the analytic discs $A_{1}, \ldots, A_{s}$ 
of class $\mathcal{P}^{\alpha}$ are obtained as attached to $M_{1}, \ldots, M_{s}$ respectively with $A_{j}(1)=A(1)$ and with the same $w$-component as $A$ such that $\left[\partial_{r} A_{1}(1)\right], \ldots,\left[\partial_{r} A_{s}(1)\right]$ are linearly independent.

\section{Filling $\alpha$-Wedges BY DisCS IN $\mathcal{P}^{\alpha}$}

Our goal here is to show that in the context of Theorem 1.1 a family of nonsmooth analytic discs attached to $V$ fills an $\alpha$-wedge over $V$ in the sense of Definition 2.2 In fact, we obtain a slightly stronger statement: actually the family of radii of the discs fills such a wedge. We begin with an abstract lemma for families of real curves, and then show that the family of radii satisfies the assumption of the lemma.

Lemma 5.1. Let $V \subset \mathbb{R}^{l} \times\{0\} \subset \mathbb{R}^{l} \times \mathbb{R}^{m-l}$ be an open subset with Lipschitz boundary at $0 \in \partial V$. Let $\varphi:[0,1] \times \bar{V} \rightarrow \mathbb{R}^{l}$ be a map of the form

$$
\varphi(t, p)=p+t^{\alpha} a(p)+b(t, p), \quad 0<\alpha<1,
$$

with $a(\cdot)$ and $b(\cdot, p)(p \in \bar{V})$ being of class $C^{1, \gamma}$ for some $0<\gamma \leq \frac{1}{\alpha}-1$ such that $a(p) \in C_{0} V \times\{0\}, b(0, p) \equiv 0$ and $\partial_{t} b(0,0) \notin \mathbb{R}^{l} \times\{0\}$. Assume that the induced map $p \mapsto b(\cdot, p)$ between $\bar{V}$ and $C^{1, \gamma}$ is also of class $C^{1, \gamma}$. Then there exist $\varepsilon>0$, a neighborhood $U$ of 0 in $\mathbb{R}^{l} \times\{0\}$, and a submanifold $M^{\prime}$ of class $C^{1, \gamma^{2}}$ with boundary $M$ at 0 in the direction of $\partial_{t} b(0,0)$ such that $\varphi$ defines a homeomorphism between $(0, \varepsilon) \times(V \cap U)$ and an $\alpha$-wedge over $V$ at 0 in $M^{\prime}$.

Proof. After a linear change of coordinates $(x, y) \in \mathbb{R}^{l} \times \mathbb{R}^{m-l}$, we may assume that $\partial_{t} b(0,0) \in 0 \times \mathbb{R}_{+}^{m-l}$. Then the map

$$
\widetilde{\varphi}(\tau, p):=\left(\varphi_{1}\left(\tau^{1 / \alpha}, p\right), \ldots, \varphi_{l}\left(\tau^{1 / \alpha}, p\right),\left(\varphi_{l+1}\left(\tau^{1 / \alpha}, p\right)\right)^{\alpha}, \ldots,\left(\varphi_{m}\left(\tau^{1 / \alpha}, p\right)\right)^{\alpha}\right)
$$

is well-defined for small $\tau \geq 0$ and $p \in \bar{V}$ close to 0 . We claim that $\tilde{\varphi}$ is $C^{1, \gamma^{2}}$ in a neighborhood of $(0,0)$ in $[0,1] \times \bar{V}$. Indeed, the claim is nontrivial only for the components $\left.\varphi_{l+j}\left(\tau^{1 / \alpha}, p\right)\right)^{\alpha}, j=1, \ldots, m-l$. Arguing as in (6), we can write $\varphi_{l+j}(t, p)=t \widehat{\varphi}(t, p)$ with $\widehat{\varphi} \in C^{\gamma}$ and $\widehat{\varphi}(0,0) \neq 0$. Then we have

$$
\begin{aligned}
\partial_{\tau}\left(\left(\varphi_{l+j}\left(\tau^{1 / \alpha}, p\right)\right)^{\alpha}\right)=\left(\varphi_{l+j}\left(\tau^{1 / \alpha}, p\right)\right)^{\alpha-1} & \left(\partial_{t} \varphi_{l+j}\right)\left(\tau^{1 / \alpha}, p\right) \tau^{(1 / \alpha)-1} \\
= & \left(\widehat{\varphi}_{l+j}\left(\tau^{1 / \alpha}, p\right)\right)^{\alpha-1}\left(\partial_{t} \varphi_{l+j}\right)\left(\tau^{1 / \alpha}, p\right) .
\end{aligned}
$$

Since the composition $h \circ f$ of two maps $h, f \in C^{\gamma}$ is in $C^{\gamma^{2}}$ and depends smoothly (in fact linearly) on $h$, it follows that the map $p \mapsto \partial_{\tau} \widetilde{\varphi}(\cdot, p)$ between $\bar{V}$ and $C^{\gamma^{2}}$ is of class $C^{1, \gamma}$. In particular, the map $p \mapsto \widetilde{\varphi}(\cdot, p)$ between $\bar{V}$ and $C^{\gamma^{2}}$ is also of class $C^{1, \gamma}$. We conclude that both derivatives $\partial_{\tau} \widetilde{\varphi}$ and $\partial_{p} \widetilde{\varphi}$ are in $C^{\gamma^{2}}$ with respect to $(\tau, p) \in[0,1] \times \bar{V}$, and the claim follows.

The computation shows that

$$
d \widetilde{\varphi}(0,0)[\mathbb{R} \oplus\{0\}]=\left(a(0), \partial_{t} b_{l+1}(0,0), \ldots, \partial_{t} b_{m}(0,0)\right) \mathbb{R}
$$

and $d \widetilde{\varphi}(0,0)\left[\mathbb{R} \oplus \mathbb{R}^{l}\right]=\mathbb{R}^{l} \oplus \mathbb{R} \partial_{t} b(0,0)$. Then, by the rank theorem, there exists a submanifold $M^{\prime}$ of class $C^{1, \gamma^{2}}$ with boundary $M$ at 0 such that $C_{0} M^{\prime}=\mathbb{R}^{l} \oplus$ $\mathbb{R}_{+} \partial_{t} b(0,0)$ and, for some $C>0$, the subset

$$
\widetilde{V}^{\prime}:=\left\{x \in M^{\prime}: \operatorname{dist}(x, V)<C \operatorname{dist}(x, \partial V)\right\}
$$


is contained in the image $\widetilde{\varphi}((0,1] \times \bar{V})$. Then the subset

$$
V^{\prime}:=\left\{x \in \mathbb{R}^{l} \times \mathbb{R}_{+}^{m-l}:\left(x_{1}, \ldots, x_{l}, x_{l+1}^{\alpha}, \ldots, x_{m}^{\alpha}\right) \in \tilde{V}^{\prime}\right\}
$$

contains an $\alpha$-wedge over $V$ at 0 satisfying the required conditions.

We now apply Lemma 5.1 to analytic discs in $\mathcal{P}^{\alpha}$. In view of Lemma 4.4, we immediately obtain:

Proposition 5.2. Let $M \subset \mathbb{C}^{n}$ be a generic submanifold of class $C^{2}, V \subset M$ an open subset with Lipschitz boundary at a point $p_{0} \in \bar{V}, 1 / 2<\alpha<1$, and let $\gamma$ be as in Lemma 5.1. Choose a family $p \mapsto A_{p} \in \mathcal{P}^{\alpha}$ of class $C^{1, \gamma}$ of attached analytic discs to $M$ for $p \in M$ in a neighborhood of $p_{0}$ such that $A_{p}(1) \equiv p$ and the following hold:

(i) the vector $\left(A_{p_{0}}\right)_{0}$, chosen according to (23) for $A=A_{p_{0}}$, is contained in $C_{p_{0}} V ;$ and

(ii) $A_{p_{0}}$ is transversal to $M$ at 1 , i.e. $\left[\partial_{r} A_{p_{0}}(1)\right] \neq 0 \in\left(T_{M} \mathbb{C}^{n}\right)_{p_{0}}$.

Then there exist $\varepsilon>0$, a neighborhood $U$ of $p_{0}$ in $M$, and a submanifold $M^{\prime}$ of class $C^{1, \gamma^{2}}$ with boundary $M$ at $p_{0}$ in the direction of $\left[\partial_{r} A_{p_{0}}(1)\right]$ such that the map $(\tau, p) \mapsto A_{p}(\tau)$ defines a homeomorphism between $(1-\varepsilon, 1) \times(V \cap U)$ and an $\alpha$-wedge $V^{\prime}$ over $V$ at $p_{0}$ in $M^{\prime}$.

\section{BAOUENDI-TREVES TYPE APPROXIMATION FOR SECTORS}

We call a generic submanifold $V \subset \mathbb{C}^{n}$ a Baouendi-Treves submanifold if, for every $s=0,1, \ldots, \infty, \omega$, every CR-function on $V$ of class $C^{s}$ can be uniformly approximated by holomorphic polynomials on compacta in $V$ in the $C^{s}$ topology. Recall that a sequence converges in the $C^{\infty}$ topology if it converges in each $C^{k}$ norm $\|f\|_{k}$ for $0 \leq k<\infty$, and that it converges in the $C^{\omega}$ topology if it converges with respect to the pseudonorm $\sup _{k} \frac{\varepsilon^{k}}{k !}\|f\|_{k}$ for some $\varepsilon>0$.

In [T95] Tumanov observed that the original proof of the BAOUEndi-Treves approximation theorem [BT81] can be adapted to the situation of a submanifold with generic edge. However, the situation considered in this paper, where the edge is not generic, is more delicate. Here we show that neighborhoods of certain sectors in $V$ are Baouendi-Treves submanifolds.

Theorem 6.1. Let $M \subset \mathbb{C}^{n}$ be a generic submanifold and $V \subset M$ an open subset with Lipschitz boundary at $p=0 \in \partial V$. Suppose that for some $v \in \mathbb{C}^{n}$ and $0<\alpha<1$, the sector $S_{v}(\alpha):=\left\{\tau^{\alpha} v: \operatorname{Re} \tau \geq 0\right\}$ is contained in $C_{p} V$. Then there exists $\varepsilon_{0}>0$ such that the open subset

$$
\left\{z \in M: \operatorname{dist}\left(z, S_{v}(\alpha)\right)<\varepsilon_{0} \operatorname{dist}(z, p)<\varepsilon_{0}^{2}\right\}
$$

is a Baouendi-Treves submanifold in $\mathbb{C}^{n}$.

We give a brief description of the method of Baouendi-Treves BT81 (see also T96 and the book [BER99|). The method is based on the convolution of a given CR-function with the differential form $e^{-\sum_{j} z_{j}^{2}} d z_{1} \wedge \cdots \wedge d z_{n}$ along a maximally totally real submanifold $N \subset M$. (Recall that a submanifold $N \subset M$ is maximally totally real if $\left(T_{p} N \cap T_{p}^{c} M\right) \cap i\left(T_{p} N \cap T_{p}^{c} M\right)=0$ and $T_{p} N+T_{p}^{c} M=T_{p} M$ for all $p \in N$.) Then, an application of the Stokes theorem shows that the convolution does not change if the submanifold is slightly deformed. Denote by $B_{0}^{s}$ and $B^{s}$ the 
open and closed unit balls in $\mathbb{R}^{s}$ respectively. The assumption required for this method to work can be stated as follows.

Lemma 6.2. Let $M \subset \mathbb{C}^{n}$ be a generic submanifold of CR-dimension $m$. Set $d:=n-m$. Suppose that for any compact $K \subset M$, there exist a holomorphic nondegenerate quadratic form $\omega(z)$ in $\mathbb{C}^{n}$ and a smooth map $\varphi: B^{n} \times B^{d} \rightarrow M$ such that the following hold:

(i) The image $\varphi\left(B_{0}^{n} \times B_{0}^{d}\right)$ contains $K$.

(ii) For each $y \in B^{d}$, the restriction $\varphi(\cdot, y)$ is an embedding of $B^{n}$ into $M$ as a maximally totally real submanifold $N_{y}$ (with boundary) such that the restriction $\left.\operatorname{Re} \omega\right|_{N_{y}}$ is positive definite.

(iii) $\varphi(x, \cdot)=$ const for every $x \in \partial B^{n}$; in particular, the boundaries of the $N_{y}$ 's are the same for all $y \in B^{d}$.

Then $M$ is a Baouendi-Treves submanifold.

Proof. Since the argument follows exactly the line of the proof given in [T96], we only give a sketch. For a CR-function $f$ on $M$ and $y \in B^{d}$, define the sequence of entire functions

$$
f_{l, y}(z):=\left(\frac{l}{\pi}\right)^{n / 2} \int_{N_{y}} f(\zeta) e^{-l \omega(\zeta-z)} d \zeta_{1} \wedge \cdots \wedge d \zeta_{n}, \quad l=1,2, \ldots
$$

The positivity condition for Re $\left.\omega\right|_{N_{y}}$ implies that $f_{l, y}$ converges to $f$ as $l \rightarrow \infty$, uniformly on compacta in the interior of $N_{y}$. It is easy to see, by using (i), that the convergence is uniform on $K$. Moreover, it follows from the fact that $f$ is CR, from (iii) and from the Stokes theorem that the functions $f_{l, y}$ are independent of $y \in B^{d}$. Thus, $f_{l, y}$ is a sequence of entire functions that converges uniformly to $f$ on $K$. It remains to approximate entire functions by holomorphic polynomials, e.g. by taking their Taylor polynomials.

In order to apply Lemma 6.2 to our situation, we have to construct the family $\varphi$. The hardest part of this construction is to satisfy the positivity condition on $\left.\operatorname{Re} \omega\right|_{N_{y}}$.

For any $\varepsilon>0$, define the real convex cone

$$
A_{\varepsilon}:=\left\{x_{1}^{2}>\frac{\varepsilon}{2}\left(x_{2}^{2}+\cdots+x_{n}^{2}\right)\right\} \subset \mathbb{R}^{n} \subset \mathbb{C}^{n}
$$

and the ball

$$
B_{\varepsilon}:=\left\{i y: y_{1}^{2}+\cdots+y_{n}^{2}<\frac{1}{1+\varepsilon}\right\} \subset i \mathbb{R}^{n} \subset \mathbb{C}^{n} .
$$

Define the "diamond" $\widetilde{A}_{\varepsilon}:=\left(-1+A_{\varepsilon}\right) \cap\left(1-A_{\varepsilon}\right) \subset \mathbb{R}^{n}$, where 1 stands for the point $(1,0, \ldots, 0) \in \mathbb{C}^{n}$. Finally, for any $i y \in B_{\varepsilon}$, let $C_{\varepsilon}(y) \subset \mathbb{C}^{n}$ be the union of all real line segments connecting $i y$ with boundary points of $\widetilde{A}_{\varepsilon}$, and let $C_{\varepsilon}$ be the union of the subsets $C_{\varepsilon}(y)$ for $i y \in B_{\varepsilon}$.

Lemma 6.3. For any $\varepsilon>0$ and any $i y \in B_{\varepsilon}$, the standard form $\operatorname{Re} \omega=\operatorname{Re} \sum_{j} z_{j}^{2}=$ $\sum_{j}\left(x_{j}^{2}-y_{j}^{2}\right)$ is positive on tangent vectors to $C_{\varepsilon}(y)$. Moreover, for any $1 \leq d \leq n$ and $\delta>0$, there exist a smooth map $\varphi: B^{n} \times B^{d} \rightarrow \mathbb{R}^{n} \oplus i \mathbb{R}^{d}$ satisfying conditions

(ii) and (iii) in Lemma 6.2 with $\omega$ as above and such that

$$
C_{\varepsilon} \cap\left(\mathbb{R}^{n} \oplus i \mathbb{R}^{d}\right) \subset \varphi\left(B_{0}^{n} \times B_{0}^{d}\right) \subset(1+\delta) C_{\varepsilon} \cap\left(\mathbb{R}^{n} \oplus i \mathbb{R}^{d}\right) .
$$


Proof. Any tangent vector $v$ to $C_{\varepsilon}\left(y_{0}\right)$ at a point $z=x+i y$ is a sum $v_{1}+v_{2}$, where $v_{1}$ is tangent to the segment connecting $z$ with a boundary point $a \in \partial \widetilde{A}_{\varepsilon}$ and $v_{2}$ is tangent to $\partial \widetilde{A}_{\varepsilon}$ at $a$. If $v_{1}=0$, the claim is clear, since $\operatorname{Re} \omega$ is positive on $\mathbb{R}^{n} \subset \mathbb{C}^{n}$. Otherwise, by rescaling $v$, we may assume $v_{1}=a-i y$. By the convexity of $\widetilde{A}_{\varepsilon}$, $a+v_{2} \notin \widetilde{A}_{\varepsilon}$. Then, by the construction of $A_{\varepsilon}$ and $B_{\varepsilon}$, we have $|y|<\left|a+v_{2}\right|$. Since $v=a+v_{2}-i y$, this shows that $\operatorname{Re} \omega(v)>0$, as required.

For the second statement, observe that $C_{\varepsilon}(y)$ can be written as $C_{\varepsilon}(y)=\{x+$ $\left.i \xi(x) y: x \in \widetilde{A}_{\varepsilon}\right\}$ for a suitable continuous function $\xi(x)$. We replace $\xi$ by a smooth function $\widetilde{\xi}$ that approximates $\xi$ in the $C^{1}$ norm such that the submanifold $\widetilde{C}_{\varepsilon}(y):=\left\{x+i \widetilde{\xi}(x) y: x \in \widetilde{A}_{\varepsilon}\right\}$ still satisfies the above positivity condition. It remains to choose $\varphi(x, y):=\sqrt{1+\delta}(x+i \widetilde{\xi}(x) y)$.

Proof of Theorem 6.1. Denote by $d$ the codimension of $M$ in $\mathbb{C}^{n}$. Without loss of generality, $p=0$. The proof will depend on whether $\alpha$ is larger or smaller than $1 / 2$. Suppose first that $\alpha>1 / 2$. Then $\varepsilon>0$ can be chosen such that, for $y:=(1+\varepsilon)^{-1 / 2}(1,0, \ldots, 0)$, the intersection $I:=C_{\varepsilon}(y) \cap(\mathbb{C} \times\{0\})$ has the angle $\alpha$ at the point $i y$. Then, for any $\lambda>0$, there exists a complex affine automorphism $F_{\lambda}: \mathbb{C}^{n} \rightarrow \mathbb{C}^{n}$ sending $i \lambda y$ to $0, \lambda C_{\varepsilon}(y) \cap(\mathbb{C} \times\{0\})$ to $S_{v}(\alpha)$ and $\lambda C_{\varepsilon} \cap\left(\mathbb{R}^{n} \oplus i \mathbb{R}^{d}\right)$ into the interior of $C_{p} V$. It follows from the definition of $C_{p} V$ that for $\lambda>0$ sufficiently small, the map $F_{\lambda}$ can be approximated on the closure $\overline{\lambda C_{\varepsilon}}$ in the $C^{1}$ norm by a diffeomorphism $\widetilde{F}_{\lambda}: \mathbb{C}^{n} \rightarrow \mathbb{C}^{n}$ sending $\lambda C_{\varepsilon} \cap\left(\mathbb{R}^{n} \oplus i \mathbb{R}^{d}\right)$ into the closure $\bar{V} \subset M$ such that $\widetilde{F}_{\lambda}(i \lambda y)=0$ and $d \widetilde{F}_{\lambda}(i \lambda y)=d F_{\lambda}(i \lambda y)$. We can now use $\widetilde{F}$ to transfer the standard form $\omega$ and the family $\varphi$ constructed in Lemma 6.3 to the image $U:=\widetilde{F}_{\lambda}\left(\lambda C_{\varepsilon} \cap\left(\mathbb{R}^{n} \oplus i \mathbb{R}^{d}\right)\right) \subset \bar{V}$ in order to have data satisfying the assumptions of Lemma 6.2 Then Lemma 6.2 yields the required conclusion.

The proof for $\alpha \leq 1 / 2$ is analogous to that in the first case $\alpha>1 / 2$, with the only exception that the above map $F_{\lambda}$ must be chosen to send the point $(\lambda, 0, \ldots, 0) \in$ $\lambda C_{\varepsilon}$ instead of $i \lambda y$ into $p=0$.

\section{Proofs of Theorems 1.11 .4}

Proof of Theorem 1.4. We first prove statement (ii). Let $A, p, q$ and $V^{\prime}$ be as in Theorem 1.4 (ii). Hence $A$ belongs to a class $\mathcal{P}^{\alpha}$ for a suitably chosen fixed $0<\beta \leq$ $2 \alpha-1$. (Recall that we fixed such $\beta$ in the definition of $\mathcal{P}^{\alpha}$.) By Proposition 4.1 if $A$ is sufficiently small, it can be extended to a family $x \mapsto A_{x} \in \mathcal{P}^{\alpha}$ of class $C^{2}$ of analytic discs attached to $M$ for $x \in M$ in a neighborhood of 0 such that $A_{0}=A$ and $A_{x}(1)=x$ for all $x$. It follows from the definition of $C_{p} V$ and the assumptions that the family $x \mapsto A_{x}$ can be chosen such that $A_{x}(\partial \Delta) \subset V$ for $x \in V$ sufficiently close to $p$. By Corollary 4.7, after an arbitrarily small (in the $C^{4}$ norm) deformation of $M$ inside $V^{\prime}$, we may assume that $A=A(\tau)$ is transversal at $\tau=1$ to $M$, i.e. $\left[\partial_{r} A(1)\right] \neq 0 \in\left(T_{M} \mathbb{C}^{n}\right)_{p}$. Moreover, given several submanifolds $V_{1}^{\prime}, \ldots, V_{s}^{\prime}$ as in Theorem 1.4 (ii), Corollary 4.7 yields $s$ linearly independent directions for the corresponding discs. Then, if $\varepsilon$ and $U$ are given by Proposition 5.2, the map $(\tau, x) \mapsto A_{x}(\tau)$ defines a homeomorphism between $(1-\varepsilon, 1) \times(V \cap U)$ and an $\alpha$-wedge $V^{\prime \prime}$ over $V$ at 0 in a submanifold $M^{\prime \prime}$ with boundary $M$ at 0 of class $C^{1, \delta}$ with $0<\delta<1$ suitably chosen. Finally, if $\varepsilon_{0}$ is given by Theorem 6.1 with $v$ as in Theorem 1.4 and if $A$ is sufficiently small, the boundary of each $A_{x}$ is contained in the set (27) for any $x \in V$ sufficiently close to $p$. Hence, for any such $x=p_{0}$, any CR-function $f$ on $V$ can be uniformly approximated by a sequence of 
polynomials in a neighborhood of the compact set $A_{p_{0}}(\partial \Delta) \subset V$. By the maximum principle, the sequence of polynomials converges uniformly on the images $A_{p}(\partial \Delta)$ for $x \in V$ sufficiently close to $p_{0}$ to a limit which defines a CR-extension of $f$ to a neighborhood of $A_{p_{0}}((1-\varepsilon, 1))$ in $V^{\prime \prime}$. Moreover, any such sequence of polynomials yields the same limit function. Summarizing, we obtain a covering of $V \cap U$ by open subsets $V_{j}$ such that $f$ extends to be CR on the interior of the subset

$$
V_{j}^{\prime \prime}:=\left\{A_{x}(\tau): x \in V_{j}, \tau \in(1-\varepsilon, 1)\right\} \subset V^{\prime \prime}
$$

for each $j$. Moreover, we can choose the covering $\left\{V_{j}\right\}$ sufficiently small so that, whenever $V_{j} \cap V_{k} \neq \emptyset$, there exists a sequence of polynomials as above that converges uniformly on the union $V_{j}^{\prime \prime} \cup V_{k}^{\prime \prime}$. By the uniqueness property of the limit, the CRextensions of $f$ to $V_{j}^{\prime \prime}$ and $V_{k}^{\prime \prime}$ coincide on the intersection. Thus we obtain a well-defined CR-extension of $f$ to $V^{\prime \prime}$, and the proof of part (ii) of Theorem 1.4 is complete.

For the proof of part (i), observe that, given a wedge $V^{\prime} \subset \mathbb{C}^{n}$ with edge $V$ at $q$, we can choose submanifolds $V_{1}^{\prime}, \ldots, V_{d}^{\prime} \subset \mathbb{C}^{n}$ of class $C^{4}$ with boundary $V$ at $q$ in $d$ linearly independent directions in $T_{q} \mathbb{C}^{n} / T_{q} M$, where $d$ denotes the codimension of $M$ in $\mathbb{C}^{n}$. By part (ii), we obtain CR-extension of any CR-function $f$ on $V \cup\left(\bigcup_{j} V_{j}^{\prime}\right)$ to $\alpha$-wedges $V_{1}^{\prime \prime}, \ldots, V_{d}^{\prime \prime}$ over $V$ at $p$ in submanifolds $M_{1}^{\prime \prime}, \ldots, M_{d}^{\prime \prime}$ respectively with boundary $M$ whose directions in $T_{p} \mathbb{C}^{n} / T_{p} M$ are also linearly independent. Then near each point $p_{0} \in V$ sufficiently close to 0 we are in the setting of the theorem of Ajrapetyan and Henkin AH81, which yields holomorphic extension of $f$ to a wedge $W_{p_{0}}$ with edge $V$ at $p_{0}$ whose direction cone can be assumed to be any cone smaller than the convex linear span of the directions of $M_{1}^{\prime \prime}, \ldots, M_{d}^{\prime \prime}$ at $p_{0}$. Recall that a subcone $C$ of an open cone $C^{\prime}$ is called smaller if the closure of $C$ intersected with the unit sphere is contained in $C^{\prime}$. In order to obtain an $\alpha$-wedge over $V$ as required, we need to estimate the size of $W_{p_{0}}$ as $p_{0}$ approaches the boundary $\partial V$. This can be done by inspecting the proof of Theorem 4.1 in [T96] and using [T93, Theorem 1.2] to obtain the required regularity of the solution of the Bishop's equation. More precisely, the first step is to obtain a deformation version of the Ajrapetyan-Henkin theorem, for which the reader is referred to [MMZ02, Proposition 3.3]. The second step is to use linear rescaling, i.e. linear maps $z \mapsto \lambda z$, to show that the size of the wedge $W$ in the Ajrapetyan-Henkin theorem is proportional to the size of the given submanifolds. Since each $V_{j}^{\prime \prime}$ is an $\alpha$-wedge over $V$ at 0 , its size near $p_{0}$ in all directions can be estimated from below by $\operatorname{dist}\left(p_{0}, \partial V\right)^{1 / \alpha}$ up to a constant. Hence also the size of $W_{p_{0}}$ has a proportional estimate from below. Now it follows directly from Definition 2.2 that the wedges $W_{p_{0}}$ cover an $\alpha$-wedge $V$ over $V^{\prime \prime}$ at 0 . Furthermore, by choosing $W_{p_{0}}$ in a suitable way and using the uniqueness of a holomorphic extension of functions into wedges, we obtain the required holomorphic extension of $f$ to $V^{\prime \prime}$. The proof of Theorem 1.4 is complete.

Proof of Theorems 1.2 and 1.3. Without loss of generality, $p=0$. As in [994. pp. 17-18], we may approximate $\gamma$ by a chain of arbitrarily small analytic discs $A_{j}$, $1 \leq j \leq l$, attached to $M$ such that, $A_{j}(\partial \Delta) \cap A_{j+1}(\partial \Delta) \neq \emptyset$ for $1 \leq j \leq l-1$, $A=A_{1}$ satisfies the assumptions of Theorem 1.4, and all other discs $A_{j}$ are $C^{1, \beta}$. By repeating the arguments of [T94], one can see that the discs $A_{j}$ can be chosen such that $A_{l}(\partial \Delta)$ has points arbitrarily close to $\gamma(1)$. In particular, if $V^{\prime}$ is given as in Theorem 1.4 it becomes a submanifold with boundary $M$ at some point of $A_{l}(\partial \Delta)$. The conclusion of Theorem 1.2 follows now from Theorem 1.4 (i) and the 
propagation of wedge extendibility along analytic discs due to TrÉPreAu [Tr90] and Tumanov [T94]. The conclusion of Theorem 1.3 follows analogously from Theorem 1.4 (ii).

Proof of Theorem 1.1. By the definition of the complex angle, there exists a CRcurve $\gamma$ satisfying the assumptions of Theorem[1.2. Then the required conclusion follows immediately from Theorem 1.2 .

\section{REFERENCES}

[AH81] Airapetyan, R.A.; Henkin, G.M. - Analytic continuation of CR-functions through the "edge of the wedge". Sov. Math., Dokl. 24 (1981), 129-132; translation from Dokl. Akad. Nauk SSSR 259 (1981), 777-781. MR 82k:32039

[BER99] Baouendi, M.S.; Ebenfelt, P.; Rothschild, L.P. - Real Submanifolds in Complex Space and Their Mappings. Princeton Math. Series 47, Princeton Univ. Press, 1999. MR 2000b:32066

[BRT94] Baouendi, M.S.; Rothschild, L.P.; Trépreau, J.-M. — On the geometry of analytic discs attached to real manifolds. J. Differential Geom. 39 (1994), 379-405. MR 95a:32026]

[BT81] Baouendi, M.S.; Treves, F. - A property of the functions and distributions annihilated by a locally integrable system of complex vector fields. Ann. Math. (2) 114, 387-421, (1981). MR 82i:35057

[BZ01] Baracco, L; Zampieri, G. - Analytic discs and extension of CR functions. Compositio Math. 127 (2001), no. 3, 289-295. MR 2002f:32064

[Bi65] Bishop, E. - Differentiable manifolds in complex Euclidean space. Duke Math. J. 32 (1965), 1-21. MR 34:369

[Bo91] Boggess, A. - CR Manifolds and the Tangential Cauchy-Riemann Complex. Studies in Advanced Mathematics. CRC Press. Boca Raton Ann Arbor Boston London 1991. MR 94e:32035

[Bo98] Boggess, A. - The holomorphic extension of $C^{k}$ CR functions on tube submanifolds. Complex analysis and applications (Warsaw, 1997). Ann. Polon. Math. 70 (1998), 3542. MR 99j:32010

[Bo99] Boggess, A. - The holomorphic extension of $H^{p}$-CR functions on tube submanifolds. Proc. Amer. Math. Soc. 127 (1999), no. 5, 1427-1435. MR 99h:32012

[BP85] Boggess, A.; Pitts, J. - CR extension near a point of higher type. Duke Math. J. 52 (1985), 67-102. MR 86k:32012

[BP82] Boggess, A.; Polking, J.C. - Holomorphic extension of CR functions. Duke Math. J. 49, 757-784 (1982). MR 84j:32014

[EG01] Eastwood, M.C.; Graham, C.R. - An Edge-of-the Wedge Theorem for Hypersurface CR Functions. J. Geom. Anal. 11 (2001), no. 4, 589-602. MR 2002i:32033

[EG02] Eastwood, M.C.; Graham, C.R. — Edge of the Wedge Theory in Hypo-Analytic Manifolds, preprint (2001), http://arXiv.org/abs/math.CV/0107183.

[HS82] Hanges, N.; Sjöstrand, J. - Propagation of analyticity for a class of nonmicrocharacteristic operators. Ann. of Math. (2) 116 (1982), no. 3, 559-577. MR 85g:58085

[HT83] Hanges, N.; Treves, F. - Propagation of holomorphic extendability of CR functions. Math. Ann. 263 (1983), no. 2, 157-177. MR 85c:58102

[HT78] Hill, C.D.; Taiani, G. - Families of analytic discs in $C^{n}$ with boundaries on a prescribed CR submanifold. Ann. Scuola Norm. Sup. Pisa Cl. Sci. (4), 5 (1978), no. 2, 327-380. MR 80c:32023

[L00] Lanza de Cristoforis, M. - Differentiability properties of an abstract autonomous composition operator. J. London Math. Soc. (2), 61 (2000), 923-936. MR 2001c:47069

[MMZ02] Meylan, F.; Mir, N.; Zaitsev, D. Analytic regularity of CR-mappings. Math. Res. Lett. 9 (2002), no. 1, 73-93. MR 2003d:32041

[Tr90] Trépreau, J.-M. - Sur la propagation des singularités dans les variétés CR. Bull. Soc. Math. France 118 (1990), no. 4, 403-450. MR 92b:58229

[T88] Tumanov, A.E. - Extension of CR-functions into a wedge from a manifold of finite type. Mat. Sb. (N.S.) 136 (178) (1988), no. 1, 128-139; translation in Math. USSR-Sb. 64 (1989), no. 1, 129-140. MR 89m:32027 
[T90] Tumanov, A.E. - Extension of CR-functions into a wedge. Mat. Sb. 181 (1990), no. 7, 951-964; translation in Math. USSR-Sb. 70 (1991), no. 2, 385-398. MR 91f:32010

[T93] Tumanov, A.E. - On the propagation of extendibility of CR functions. Complex analysis and geometry (Trento, 1993), 479-498, Lecture Notes in Pure and Appl. Math., 173, Dekker, New York, 1996. MR 96j:32012

[T94] Tumanov, A.E. - Connections and propagation of analyticity for CR functions. Duke Math. J. 73 (1994), no. 1, 1-24. MR 95i:32025

[T95] Tumanov, A.E. - Propagation of extendibility of CR functions on manifolds with edges. Multidimensional complex analysis and partial differential equations (Sao Carlos, 1995), 259-269, Contemp. Math. 205, Amer. Math. Soc., Providence, RI, 1997. MR 98e:32015

[T96] Tumanov, A.E. - Analytic discs and the extendibility of CR functions. Integral geometry, Radon transforms and complex analysis (Venice, 1996), 123-141, Lecture Notes in Math. 1684, Springer, Berlin, 1998. MR 99f:32024

[ZZ01] Zaitsev, D; Zampieri, G. - Extension of CR-functions on wedges. Math. Ann., to appear.

School of Mathematics, Trinity College, Dublin 2, Ireland

E-mail address: zaitsev@maths.tcd.ie

Dipartimento di Matematica, Università di Padova, via Belzoni 7, 35131 Padova, Italy

E-mail address: zampieri@math.unipd.it 